\title{
De la República de las Letras a Internet: de la Ciudad Letrada a la cibercultura y las tecnologías del S. XXI
}

\author{
ELOY MARTOS NÚÑEZ \\ Universidad de Extremadura \\ España
}

Resumen. Describe la evolución del concepto de la "República de las Letras" hacia un concepto más omnicomprensivo y tecnológico de "Ciudad Letrada" y sus implicaciones en los campos educativo y cultural. Se examinan igualmente otras controversias sobre el sentido de dichos conceptos aplicados a los contextos actuales y, en particular, en relación a Internet y los nuevos fenómenos de la sociedad de la información.

Palabras clave: literacidad, internet, República de las letras, cultura letrada, TIC.

\begin{abstract}
This article attempts to show the evolution of the concept of Republic of the Letters towards a more global concept of Learned City and its implications in the fields of educational and cultural. It also examines other disputes about the meaning of these concepts applied to current contexts and, in particular, the internet and new phenomena of information society.
\end{abstract}

Keywords: literacy, internet, Republic of the letters, literate culture, TIC. 


\section{Introducción}

El término República de las Letras fue acuñado por Pierre Bayle en 1664 en el título del periódico que él fundó, Nouvelles de la Republique des Lettres. Si bien hay precedentes de esta concepción en épocas precedentes (por ejemplo, en la Universidad de París y su Colegio de Navarra), es con la Ilustración cuando cobra fuerza este ideal de comunidad intelectual universal, sin fronteras fijas y sin ubicaciones concretas (a diferencia de las Sociedades y Academias).

Por su parte, la idea de ciudad letrada es una elaboración más concreta, que se ha usado en referencia a la organización colonial y luego republicana de los países latinoamericanos, en que todo lo relacionado con la escritura ocupaba un lugar de preeminencia y se vinculaba a los centros y posiciones de poder (Jouvé, 2005). En otro sentido aún más determinado, se habla de "Ciudad de las Letras" para referirse a conjuntos arquitectónicos como bibliotecas, museos o centros culturales donde se ofrecen de forma integrada servicios, eventos, etc.

Ambos conceptos quedan subsumidos en una propuesta más ambiciosa, que es la que realiza el profesor Roger Chartier (1994) cuando habla de cultura letrada como un ámbito específico que bien podemos imaginar, al modo de P. Bayle, como una magnífica "ciudad" que puede ser recorrida y habitada, esto es, utilizada plenamente por los ciudadanos. La modernidad de estas propuestas se reavivan actualmente con la democratización de la cultura y la necesaria función social de la ciencia y la tecnología, intensificadas por el impacto de la sociedad de la información y lo que se ha denominado la nueva "cultura de la convergencia y la participación” (Jenkins, 2008).

Con todo, es preciso matizar el alcance de dichas concepciones y vislumbrar las nuevas demandas culturales para entender el dinamismo de la sociedad actual. Así, bajo el diseño de los grandes conjuntos culturales, como el Centro G. Pompidou de París, subyace la visión republicana del acceso a la cultura, pero también la construcción centralizada y casi "faraónica" de grandes complejos, que tratan de emular los "palacios de la memoria" de que hablaba San Agustín, y que suponen, en línea con lo que plantea Pascale Casanova (2001), antigua discípula de Pierre Bourdieu, un desarrollo hegemónico de una determinada estructura cultural, en detrimento precisamente de lo que la Unesco define como "culturas locales" de la alfabetización, esto es lo que se 
conoce como territorios periféricos o "menores" de la literatura, si hemos de aplicar las categorías de la teoría de los polisistemas (Even-Zohar, 1990).

Pascale Casanova describe, en efecto, los mecanismos por los que surge esta República mundial de las letras, a la luz de las teorías de Bourdieu, por ejemplo, el concepto de "capital" entendido en sentido amplio, no sólo economicista, y ligado a cómo una determinada comunidad o zona de concentración (en este caso París) pone en valor su patrimonio literario y lo proyecta como foco de irradiación. Ciertamente, todos los valores (las prácticas o el canon de lecturas) cobran sentido en su posición dentro del “campo" interno (por ejemplo, como literatura nacional o vernácula) y a su vez por la relación de ésta con las otras construcciones literarias inter o supranacionales, que son espacios que se "inventan" o se descubren según dinámicas y tensiones muy diversas.

Dicho internacionalismo literario se ve hoy suplantado por un mecanismo mucho más patente, la mundialización del comercio. Sea cual sea el foco irradiador, Nueva York u otro enclave, lo cierto es que la industria cultural, el valor económico, se ha superpuesto al valor académico. De hecho, en la primitiva República de las Letras la loa al talento individual, al valor de las ideas como algo intangible, se ve ahora sustituida por los datos mucho más prosaicos de su tasación como productos tangibles y que circulan conforme a unos canales socioeconómicos determinados; donde, por decirlo en pocas palabras, los mecanismos de control o la reproductividad técnica se plantean como los grandes problemas, más que la censura o la creación. Las textos son ya vistos, pues, como productos del mercado, igual que el papel de los lectores ha cambiado, y ahora ya tienen otras exigencias y habilidades, propias de una cultura de la convergencia y la participación (Jenkins, 2008). Y el resultado final es el que predice Casanova: la desintegración o desmantelamiento de los "capitales culturales y literarios" acumulados en los distintos espacios hegemónicos, en pro de una nueva realidad mucho más ubicua.

\section{La República de las Letras en su génesis sociohistórica}

La idea originaria de la República de las Letras se configuraba como un ideal ilustrado que pretendía la universalización del conocimiento a través del libre intercambio de ideas entre pensadores y científicos. Como dice el padre Feijoo, el ciudadano de esta República se considera "un hombre libre de la República de las 
Letras". Es, pues, un espacio o dominio abierto, sin policía ni fronteras, y sin necesidad tampoco de estar anclado en un edificio o institución concreta, como una Academia - y que por eso mismo prefigura lo que luego va a ser internet-, en el cual no hay más límites y desigualdades que las que marque el talento de cada uno. Todos, pues, estarían en pie de igualdad, todos pueden leer y escribir, formular ideas y enjuiciarlas, y las “ideas mejores”, valoradas en esta red comunitaria, terminarían por ser reconocidas.

Ciertamente, esta idea se ve favorecida por hechos relevantes de la historia cultural de la Francia ilustrada, como fue la irrupción de los llamados "salones" parisinos o la creciente importancia del "discurso epistolar", esto es, de las correspondencias entre personas "savantes". Hay al respecto un debate importante que atañe a la sociología del conocimiento (Bourdieu, 1992) y a la historiografía, pues para autores como Dena Goodman (1994) la República de las Letras no surge como un proyecto altruista de difusión de la ciencia y el pensamiento sino más bien vinculado a este nuevo medio de los salones, cafés, etc., así como a la nueva retórica influenciada por el creciente papel de estas mujeres como mediadoras ("salonnières"). Es decir, el énfasis habría que marcarlo en estas nuevas "formas de cortesía y de socialización" imperantes en los salones de París o Londres.

De hecho, cuando se habla de intercambios en el ámbito de la República de las Letras, no hay que entender sólo ideas; las correspondencias de la época y la actividad en estos salones nos ilustran todo tipo de contactos, recomendaciones, "encargos" y todo lo que hoy podríamos denominar como "intermediaciones" para, en muchos casos, procurar privilegios o posiciones de favor. De hecho, como advierte Robert Darnton (2000), lejos del funcionamiento igualitario como un ágora, la República de las Letras se enturbió precisamente por esta política de privilegios que gravitaba sobre la edición de los libros.

No obstante, la idea de P. Bayle y de otros ilustrados se acerca a la visión actual de una red de pensadores en contacto, que vienen a conformar un "enclave de libertad" al margen de las presiones de la religión o del despotismo, una especie de ciudad filosófica y de la ciencia, y, por principio, alejada de la religión. Por eso algunos autores, como Peter Gay ${ }^{1}$, argumentan que no cabe solapar las ideas de Ilustración y la de República

\footnotetext{
${ }^{1}$ Véase Berenzon, B. (1999): Historia es inconsciente. La historia cultural: Peter Gay y Robert Darnto. México
} 
de las Letras, pues para él, aquélla sería más bien el resultado del "cóctel" entre tradiciones como las de Newton y Lucrecio, esto es, el nuevo espíritu científico y la incredulidad atea.

Sea como sea, se forjó una República de las Letras de carácter transatlántico, puesto que estos periódicos franceses e ingleses influyeron en seguida en las colonias americanas, la cual fructificó en entidades como la "Athenian Society", fundada por John Dunton en 1691 y predecesora del "Tellatale Club" de Harvard, o en los Salones, como el de Philadelphia, con participación a veces de la mujer siguiendo el modelo anteriormente comentado de mujer "savante" y de sociabilidad que emula los modelos europeos. José Antonio Marina ${ }^{2}$ subraya que "la Enciclopedia aspiraba a ser el corazón de la República de las Letras, a provocar una gigantesca movilización educativa, social, política”.

La situación posterior, a tenor de la descripción de R. Darnton (1994), dejó estas ideas en una suerte de utopía o fantasía histórica, no sólo por el fracaso del proyecto ilustrado sino porque la propia evolución posterior de la sociedad capitalista cercenó esta visión; por ejemplo, se impuso una compartimentación o división de los campos científicos que ahondaba la separación entre ciencias y letras, artes y oficios, todo ello impulsado por sociedades y revistas científicas que tendían a la exclusión y a defender posiciones de status más que el conocimiento general o el bien general, lejos, pues, de la orientación didáctica y abierta de la primera República de las Letras.

Ahora bien, Habermas (1991) por su parte, ha descrito bien el concepto de "esfera pública" como un espacio vinculado al nacimiento de la sociedad burguesa. De hecho, se consideraba a los dueños de la propiedad privada como ciudadanos iguales, el acceso a esta nueva esfera pública alfabetizada, al margen del Estado, era en principio universal, si bien esta inclusión no afectaba al resto de la sociedad, a las clases subalternas; se limitaba en realidad al dominio privado y se manifestaba en círculos más o menos restringidos, con un contenido asertivo -i.e. de autoafirmación y de ejercicio de la crítica sobre la vida social- y con tendencia de crecimiento a través de las clases medias.

\footnotetext{
${ }^{2}$ http://www.elmundo.es/papel/2004/01/03/docume/1551864.html
} 
Debemos a Chartier el análisis de estas cuestiones en relación a la lectura, como cuando habla de la aparición de un nuevo espacio público en el siglo XVIII en Francia y en Inglaterra (en el marco de nuevas formas de sociabilidad ${ }^{3}$, salones, clubes, logias, sociedades literarias), pero que concernía sólo a la circulación del escrito impreso. El S. XIX también produjo nuevas discontinuidades en este proceso, por ejemplo, se agregaron como lectores nuevos públicos, las mujeres, los niños o los obreros. En todo caso, la diferencia sociológica entre "público" y "pueblo", según Chartier, viene mediada justamente por la alfabetización, es la gente iletrada la que se comporta como “chusma”, fácil de ser llevada por las pasiones o la manipulación. De ahí la conexión entre este público letrado y el nuevo ámbito sociopolítico que dio origen a la Revolución Francesa.

Ciertamente, las nuevas tecnologías y el consiguiente "vuelco" social y político de las sociedades del S. XXI respecto a sus precedentes de los siglos anteriores, obligan a reformular la República de las Letras más allá de un simple "desideratum" o de una imagen alegórica del saber. La comunidad científica actual se centra en el progreso del conocimiento, pero los promotores de la República de las Letras supeditaban éste a un fin social, a un interés general, que llevase a la "felicidad". Los neurocientíficos actuales se acercan a estos conceptos en términos más prácticos, al subrayar la ausencia de miedos y de preconcepciones como mecanismos para promover la felicidad y el conocimiento (Denté, 2007). A este propósito hay que entender la obra desmitificadora del Padre Feijoo tiene sentido a la luz de ese ideario de la República de las Letras, es decir, "difundir la luz" para combatir los errores y ayudar a los ciudadanos.

Actualmente, la irrupción de Internet y las nuevas herramientas parecen relanzar no sólo la primitiva idea de la República de las Letras -a partir de repositorios o fuentes de información de libre acceso, como Open Content Alliance, Open Knowledge Commons, OpenCourseWare, Internet Archive o Wikipedia- sino el propio espíritu de la "ciudad letrada", como encarnación de esa simbiosis entre la cultura letrada descrita por Roger Chartier y la nueva cultura cosmopolita, multicultural y participativa.

Es decir, la "ciudad letrada" en que podemos pensar será un espacio híbrido congruente con los nuevos fenómenos urbanos-, un ámbito de encuentro de prácticas

\footnotetext{
${ }^{3}$ Véase Lilti, A. (2005) Le Monde des salons : sociabilité et mondanité à Paris au XVIIIe siècle. París: Fayard.
} 
culturales "dialógicas" y "polifónicas", en el sentido de Bajtin, que plasmará esta nueva sensibilidad. El libre acceso, la difusión y la participación en el conocimiento son señas inalienables de esta nueva realidad.

En eso se diferencia de la visión de la cultura letrada tradicional, que fomentaba formas de sociabilidad restrictiva, que derivaba hacia el mundo profesional (los salones, cafés o clubes) o doméstico (el hogar como espacio de intimidad). En cambio, como hoy percibimos en internet, lo privado y lo público a menudo se solapan, recuperando esa sociabilidad anónima de la calle, de la "plaza pública", en los términos en que la describe Bajtin (1987). Internet, en efecto, sirve de escaparate, encrucijada y medio de difusión de todas estas prácticas.

\section{De la ciudad letrada a la cibercultura y las tecnologías del S. XXI}

Estamos ante unos escenarios culturales totalmente nuevos, donde los conceptos literarios y estéticos clásicos, como autor, genio, género, vanguardia, etc. se han transmutado en otras categorías, donde el peso de lo económico y de lo social es mucho más importante que este universo de temas e inquietudes que acompañaban la literatura y el arte de, por ejemplo, el S. XIX. La mercantilización de la cultura ha venido acompañada de un desarrollo tecnológico en el campo de la industria cultural y, más concretamente, en lo que con acierto se ha llamado "el capitalismo de ficción". Por ejemplo, la intermedialidad supone que el universo de ficción no se lanza ya sólo de una de las artes (la literatura, el cine, etc.) sino en productos integrados y secuenciados para producir el mayor impacto y beneficio.

En este sentido, el artefacto por excelencia de la cultura escrita, el libro, está sufriendo un proceso acelerado de obsolescencia y metamorfosis. Obsolescencia, porque el libro clásico, en su uso tradicional ${ }^{4}$, se está desgastando, está cediendo terreno, hacia formatos más "cómodos" como los digitales y multimediáticos. Metamorfosis porque, a medida que surgen todos estos nuevos dispositivos $\mathrm{y}$ "artefactos", el libro se está reinventando, bien a través de nuevos soportes, como el

\footnotetext{
${ }^{4}$ Por ejemplo, las enciclopedias de referencia que ocupaban decenas de volúmenes en aparatosos estantes, hoy ya no tienen sentido - la información contenida en ella se hacía rápidamente caduca- ni, diríamos, espacio físico.
} 
libro electrónico, o bien explorando nuevas vías, por ejemplo, el libro artístico o el dirigido a unos colectivos especializados.

La fragmentación, la llamada "cultura mosaico", la segmentación de audiencias, todo ello posibilita el afloramiento de diversas subculturas que se mueven dentro de los mecanismos de la posmodernidad, es decir, fomentando la hibridación y el reciclaje. Así, la gran fábrica de mitos modernos y de superhéroes que fue la Universal en la década de los 30 y 40, es hoy la misma fuente, debidamente actualizada y "tuneada", de grandes sagas y producciones contemporáneas (Spider Man, Batman, etc.)

El desarrollo de la cultura letrada y, más concretamente, de las vanguardias de los S. XIX y XX se asentaba en una idea subyacente de "progreso", las artes buscaban siempre el progreso, profundización o perfeccionamiento en sus lenguajes y tratamientos temáticos, es decir, el devenir estético se asemejaba a una explosión o afloramiento continuo de nuevas corrientes y tendencias.

El dinamismo de la sociedad mediática y de la cibercultura ha cambiado notablemente respecto a este modelo, primero, porque la multiculturalidad ha alterado los focos de irradiación y de poder (por ejemplo, el París del S. XIX) en el ámbito de la cultura, que ahora, mucho más mercantilizada, se transforma en una producción más errática, y que ya no sigue los dictados de una cultura académica -vinculada a las clases superiores- sino los del mercado. Este mismo es el que alienta las culturas populares y mediáticas, vinculadas precisamente a las clases subalternas, de modo que ya no se construye a partir de un mecanismo de imitación de la high culture, sino de otros resortes diversos y estratificados, "rizomáticos" incluso, pues el mismo patrón se puede “vender” en un área del Mediterráneo, Canadá, Australia, etc.

Los campos de la cultura, siguiendo a Bourdieu, serían ámbitos surgidos del conflicto y la negociación, como acertadamente indica J. L. Barbero ${ }^{5}$ :

Leer hoy es un montón de prácticas diferentes. Primero, prácticas históricas que no han desaparecido y que se superponen a las más modernas. La lectura se encuentra fragmentada. Primero: cada institución no tiene que ver con las otras; la escuela no tiene nada que ver con la biblioteca, la biblioteca no tiene nada que ver con la escuela, los intelectuales no tienen nada que ver con la lectura de la gente, la gente no tiene nada que ver con los intelectuales; la relación de los diversos sectores que tienen que ver con la

\footnotetext{
${ }^{5}$ J.L. Barbero: Los modos de leer, entrevista disponible en http://www.c3fes.net/docs/modosleerbarbero.pdf
} 
lectura es nula. Segundo: ni la oralidad, ni la cultura oral, ni la cultura de la imagen aparecen como claves del mundo de lectura. Tercero: todo lo que se habla de leer como parte de un ejercicio de participación ciudadana queda completamente anulado y no se ve ni en la escuela ni en las bibliotecas; la noción de lectura es una noción instrumental que está muy ligada a la función o bien escolar, o bien después a la función laboral.

Es cierto que una persona que hoy escucha un audiolibro está en una situación parecida a la de aquel auditorio analfabeto del S. XVII que oía en voz alta el Quijote, es decir, los dos leen de oídas. La actitud mental, la cosmovisión, los gustos incluso de ambos públicos pueden guardar muchas afinidades, pero la diferencia entre ambas culturas es sustancial, de entrada, en una cosa. Si, siguiendo el modelo del cine, inventariáramos el atrezo de una ciudad del S. XVII y de una ciudad del S. XXI, en ese hipotético "contenedor" veríamos que lo que más cambian son los "artefactos", el mundo de objetos que nos rodea. El artefacto debe ser entendido no desde un reduccionismo funcionalista, no se trata de un simple auxiliar o instrumento, sino que debe comprenderse en toda su dimensión cultural, por ejemplo, con referencia al socioconstructivismo que se apoya en las ideas de Vygotsky. El artefacto surge de la interacción y del aprendizaje compartido, por eso se puede definir como "un aspecto del mundo material con un uso recordado colectivamente" (Cole y Holland, 1995: 476).

El problema en la cibercultura y en la sociedad de consumo en general es la hipertrofia de las tecnologías para hacer más opaco lo que precisamente está detrás: las prácticas sociales que le dan sentido e intencionalidad. El artefacto no tiene sentido o intencionalidad, por más evidente que nos parezca tal o cual utensilio, es la práctica y la memoria social lo que confiere un valor de uso y de intercambio a un artefacto. Los valores de conectividad, participación, colaboración, etc., que propugna la cultura digital están impulsando una idea de "inteligencia colectiva" que tiene significativas concomitancias con otro concepto difundido por las ciencias cognitivas: la hipótesis de la mente extendida (Clark, 1998). Las redes sociales y otras formas de subculturas son ejemplo de "ambientes" construidos que pueden llegar a construir una cognición de alcance social; el mundo de los fans y las sagas es otra buena prueba de ello.

La hipótesis de un exocerebro o cerebro externo (Bartra, 2004) está también formulada dentro de los estudios neurocientíficos y se apoya en la plasticidad cerebral, es decir, en cómo el cerebro puede acudir a un circuito externo, como son los mecanismos culturales, para hacer una función cognitiva, por ejemplo, almacenar 
información. En su origen, una herramienta podía suplantar o mejorar una habilidad natural, por ejemplo, la de marcar un lugar o contar algo. Con el devenir del tiempo, el descifrar señales y el uso de la escritura para llevar las cuenta de algo que se almacena, van a llevarnos a tecnologías y a artefactos concretos, como el libro.

El ciberpunk no es más que la versión sofisticada de esta conciencia inicial de nuestra vinculación con el artefacto, o, como bien argumenta Sloterdisk (2003), de la necesidad que tenemos de convivir con nuestro entorno, esto es, con nuestros animales y también con "nuestras máquinas". La creación de un sistema simbólico, de un imaginario, de unos valores "intangibles", es lo que produce esta síntesis entre lo material y lo inmaterial. Los objetos se convierten en prótesis que usamos y repensamos continuamente.

A medida que se han desarrollado lo que Castoriadis (1999) ha llamado los imaginarios sociales (la moral, el derecho, etc.), los artefactos tradicionales conviven otro tipo de artefactos, los epistémicos, aquellos en que el pensamiento se "recrea" porque son herramientas metacognitivas, que perfeccionan y modelan la propia mente. Por ejemplo, las herramientas epistémicas que hay detrás de un mapa de conceptos son evidentes, aunque no tenga la forma de un martillo o de una pizarra.

Los "paquetes de información", en el S. XXI, son cada vez más grandes y su gestión más compleja. Se habla por eso de un lector polialfabetizado, porque para procesar toda esa información debe conocer los distintos alfabetismos. Internet, los móviles y otros dispositivos de la cultura actual, no son sólo una tecnología o una red de contactos, sino una gran prótesis, artefactos que nos permite depositar, como si fuera el exocerebro de Bartra, grandes bloques de información.

El reto es claro: la cultura mercantilizada no privilegia, como hacía la cultura letrada, la transmisión de la memoria, no necesita de un "legado" activo sino de una gran circulación de sus mercancías, y eso lo consigue gracias a las "modas". El marketing invasivo es una forma de ocultación, de exclusión de ese patrimonio cultural, sustituido por productos de consumo homogeneizados.

El acceso al libro no es sólo el acceso individual a la mercancía reproducida técnicamente, comprada, adquirida o consultada en una biblioteca: es el acceso en el sentido que antes indicábamos, de interacción social, de posibilidades de repensar, compartir, recrear, de entenderlo, como decíamos antes, como un aspecto del mundo 
material con un uso recordado colectivamente. Encontrar ese valor es independiente de su posición en el mercado, del precio, pues, recordando el adagio machadiano, "todo necio/ confunde valor y precio".

La República de las Letras está en esa encrucijada que veíamos en la cita de J. L. Barbero. Más que a una explosión de culturas, asistimos a una implosión, con materiales que se derriban "hacia adentro", con una "voladura" más o menos controlada, y en la que, como en algunos videojuegos, tenemos que movernos rápidos para escapar de los "cascotes" que caen. Como en Farenheit 451, el objetivo final tal vez sea crear un "solar vacío" de cultura letrada, en el sentido más noble, pero lleno de artefactos, modas y mercancías de todo tipo. La alfabetización parece omnipresente, a juzgar por la cantidad de lenguajes, soportes y dispositivos que nos inundan, pero a la vez parece ausente, si tenemos en cuenta la escasa pericia que se espera y se fomenta en sus potenciales usuarios.

El reto sigue estribando, por tanto, en superar este "fetichismo" propio de algunos segmentos cada vez más numerosos, como los llamados geek, hackers o nerds, también descritos como neófilos (gusto por lo nuevo) y tecnófilos, adictos a la tecnología y la informática. El escenario ideal geek, por ejemplo, es un ciber, un campus party, es decir, lo contrario a una sala de debates, con libros, etc., aunque ello no impida que puedan tener entre ellos prácticas colaborativas. Internet se erige en el centro de la vida de estas personas, que se vuelven consumidores compulsivos de estas tecnologías y de todos sus más novedosos productos. Todas estas manifestaciones tienen el denominador común de la llamada cultura friki, esto es, de quienes son aficionados o fans de algo y lo llevan hasta sus últimas consecuencias, ya que suelen hacer las cosas que hacen por diversión y no por interés Es lo que vemos en los fanfics, dentro de la llamada cultura del fan fiction, los aficionados recrean textos de las obras y sagas favoritas, y no lo hacen compitiendo en premios o por dinero. En este sentido, su conducta se parece a la de los librepensadores que impulsaron los primeros tiempos de la República de las Letras ${ }^{6}$. De hecho, los defensores del software libre se ven a sí mismos como herederos de esta tradición puesto que el uso de las nuevas tecnologías potenciará, según ellos, la libre circulación de conocimiento.

\footnotetext{
${ }^{6}$ Véase "Geek definition" en http://koeln.ccc.de/ablage/artikel/geek-definition.xml
} 
Otros problemas, como el equilibrio entre los derechos de autor y el uso en la red de estos materiales, están creando debates donde el elemento económico está más presente que nunca. Pero el auténtico problema sigue siendo que si bien la población letrada, en un sentido abarcador y que comprendía los sectores populares alfabetizados y no simplemente el vulgo- era la base de este proyecto primitivo de la República de las Letras, en la actualidad se favorece esto que Tomás Cuesta ${ }^{7}$ ha llamado, con cierta gracia, "muertos ilustres y vivos iletrados". La cultura letrada se presenta, así, como algo museístico, pasado, "muerto", y el futuro como un escenario de cables, aparatos e informática, en la línea más ciberpunk.

Reflotar el proyecto de República de las Letras supone, primero, repensar una nueva síntesis entre las Humanidades y las Ciencias Sociales, por una parte, y la Ciencia y la Tecnología, por la otra. Mientras tanto, internet se ha convertido en el núcleo del ocio de los jóvenes, ha amparado nuevas formas de sociabilidad y ha generado una convergencia de lenguajes sin precedentes. Si el retablo en el Barroco es la expresión de una cosmovisión religiosa compleja, el retablo politemático de internet sería también la expresión de la mente híbrida y de todas las formas y culturas plurales que la habitan, que ya no se subordinan a una composición central, que da estabilidad, sino más bien a una composición en espiral, rizomática, que produce patrones semejante en distintos ámbitos, llámese una cultura local, nacional o transnacional. En efecto, hoy es posible una República activa de las Letras desde cualquier localidad del globo, por humilde que sea, precisamente gracias a estas tecnologías que permiten la conectividad y el acceso a la información.

Los otros pilares no son tecnológicos sino culturales. Ya que el hipertexto no se presenta habitualmente con la figura de ningún mediador, es decir, ya que la cultura letrada tenía diversos mediadores especializados, desde el profesor al bibliotecario, el librero o los propios padres que tutelaban las lecturas, se impone la necesidad de formar tecnológicamente no sólo en las habilidades de manejo de la información y programas, sino justamente en aquellos aspectos depauperados que hemos visto anteriormente, el papel de la memoria y de la tradición cultural, la interacción social activa y "en presencia”, la facilitación de "claves" culturales que permitan luego filtrar y discriminar

\footnotetext{
${ }^{7}$ http://desdeelparedon.blogspot.com/2010/03/tomas-cuesta-muertos-ilustres-y-vivos.html
} 
toda la información disponible, etc. La República de las Letras se concibió también como un proyecto pedagógico y de concienciación; en aquella época, la tertulia, los salones, la correspondencia epistolar, eran las formas preferentes de sociabilidad. En el nuevo contexto, hace falta reinventar estos escenarios con fórmulas no obsoletas, por ejemplo, talleres, perfomances, etc.

A nuestro juicio, no hay que colocar el énfasis en los novedosos artefactos que han aparecido, como el libro electrónico, sino en estas nuevas formas de sociabilidad y en las prácticas culturales que alberguen instituciones que siguen siendo nucleadoras, como la familia, la escuela o los centros comerciales donde se distribuyen los productos de la cultura. Las prácticas, digamos, históricas, vinculadas a la cultura letrada, y las prácticas nuevas, vinculadas a la cibercultura y a la sociedad mediática, coexisten y se superponen presionando desde dentro con ese carácter implosivo que decíamos tiene la cultura del S. XXI. Por ejemplo, los problemas de la autoría, de la cita o del plagio y de otras cuestiones que formaban parte de un debate intelectual, se ven enseguida trasladados a otros ámbitos bastante más mercantiles, como es el de la reproductividad técnica de ese artefacto cultural producido en serie, y sus distintas alternativas: el ejemplar, la fotocopia, la descarga (i)legal, etc. El resultado para el usuario, es poder conseguir ese objeto, pero, por medio, los hilos del debate no son los de la cultura letrada sino otros mucho más economistas, legales, etc. Umbero Eco (1979) subraya que las editoriales ya saben y "amortizan" en el precio el hecho de que sus libros van a ser fotocopiados, de modo que tenemos nuevamente un debate que no es el de la República de las Letras, el de la apertura y la "explosión" del conocimiento, sino más bien el de reparto de derechos y parcelas, la "implosión", la cultura hacia dentro y como "campo" (Bourdieu, 1992) constituido socialmente y fruto de presiones, conflictos y privilegios.

La etnografía de internet está aún por hacerse, pero tampoco está exenta de todos estos conflictos que están caracterizando las relaciones entre productos en la industria y servicios culturales del S. XXI. El poder de la cultura mediática y digital, y sus modas rutilantes, son auténticas barreras, "ruido", que impide o invade una cultura mucho más abarcadora y plural, donde, como se está apreciando continuamente, la tradición clásica y las nuevas corrientes se dan la mano continuamente. La mitología, por ejemplo, es un discurso más vivo que nunca gracias a la industria de la ficción, sólo que los cibernautas desconocen las fuentes o referentes de los superhéroes o de tal o cual ficción que se ha 
convertido en éxito. Si ser librepensador en el S. XVIII suponía poder defenderse de los abusos de la autocracia y de la censura de la Iglesia, en la actualidad el librepensador debe poder ser capaz de enfrentar las prácticas y los contenidos dominantes con similar espíritu crítico.

Ciertamente, deconstruir las trampas de la civilización tecnológica y de las formas culturales dominantes es muy difícil, precisamente por la fragmentación existente y por el poder de los media. La alegoría perfecta, a nuestro juicio, es la que traza Ray Bradbury en Farenheit 451: no hace falta la policía para reprimir los libros, que no interpretamos en sentido literal sino como emblema de la cultura disidente; son simplemente los bomberos los que retiran un objeto que ha llegado a ser socialmente molesto e irrelevante, puesto que la información le viene dada por otros canales, aparentemente democráticos y bienpensantes, pero que tienden al totalitarismo. Los rebeldes, los proscritos, toman como alternativa situarse en la periferia, en la marginalidad de ese sistema y construir una "salida" que el sistema no pueda anular a los "hombres libro". ¿Quiere eso decir que Bradbury propugna una vuelta a la oralidad y a la lectura de oídas para salir del infierno en que la sociedad coloca al que quiere ser librepensador?

Yendo más allá de una lectura literal, creemos que lo que pretende Brabdury con esta fábula es lo mismo que Eco cuando reivindica el papel humanizador de la biblioteca, al servicio del ciudadano: hacer que la lectura y la escritura sean vehículos de apropiación del caudal que llamamos tradición, en el sentido más amplio (pues también es ya tradición Fellini, Picasso o los Beatles), y favorecer además que sea una práctica inclusiva y socialmente compartida, porque, como dicen los neurocientíficos, el aprendizaje surge de la interacción y no del consumo aislado o incluso narcisista con que el mercado separa al autor y a los posibles públicos. También la innovación o el cambio social exigen un ámbito de intervención, de participación. El canon letrado es la tesis, la dispersión del mercado es la antítesis, y ahora hacen falta las nuevas síntesis que concilien todas las formas plurales de cultura.

Para muchos, internet es esa nueva forma de bien comunal o "procomún" (Bollier, 2003) donde se pueden compartir contenidos y recursos, y donde caben todas las subculturas y corrientes significativas dentro del panorama cultural. Y la que recogería también esa tendencia "rizomática", descentralizada de las nuevas formas 
culturales, pues de una cultura local no es difícil que se irradien prácticas relevantes para otras y que se produzca una difusión e interacción abiertas y masivas. Lo cual era lo que preconizaba el espíritu de la República de las Letras. Los dos polos, la cultura local y la cultura virtual universal, se dan la mano de forma bajtiniana: la cultura local relativiza e invierte los valores de la sociedad mediática y nos recuerda el aquí y ahora, pero a la vez, como el aleph de Borges, es capaz de utilizar internet y sus infraestructuras como esa ventana abierta al mundo susceptible de enriquecer los problemas de la vida cotidiana.

En pocas palabras, el ideal democrático y universalizador de la República de Letras es posible, en el sentido de que es viable, con los nuevos contextos, repensar un modelo de educación que incida sobre el pensamiento crítico y las capacidades creativas de un ciudadano cada vez más polialfabetizado, capaz de integrar diferentes expresiones culturales y de utilizar multitud de lenguajes y soportes.

\section{Referencias bibliográficas}

Bajtin, M. (1974). La cultura popular en la Edad Media y el Renacimiento. El contexto de François Rabelais. Barcelona: Barral Editores.

Bartra, A. (2007). Antropología del Cerebro. La conciencia y los sistemas simbólicos. México: Fondo de Cultura Económica.

Bollier, D. (2003). El redescubrimiento del procomún. Novática: Revista de la Asociación de Técnicos de Informática, 163, 10-12

Bourdieu, P. (1992). Les regles de l'art: genèse et structure du champ littéraire. Paris:Seul.

Bremond, C. (1966). La lógica de los posibles narrativos. Comunicaciones/ Análisis estructural del relato, 87-110

Casanova, P. (2001). La república mundial de las letras. Barcelona: Anagrama

Chartier, R. (1994). El orden de los libros: lectores, autores, bibliotecas en Europa entre los siglos XIV y XVIII. Barcelona: Gedisa.

Chartier, R. (2001). Muerte o transfiguración del lector. Revista de Occidente, 23, 72-86

Clark, A. y Chalmers, D.J. (1998). The Extended Mind, Analysis 58,10-23.

Castoriadis (1999). L'institution imaginaire de la société, Paris: Editions du Seuil, collection «Points- Essais »

Darnton, R. (2000). La gran matanza de gatos y otros episodios en la historia de la cultura francesa (2a . Ed.). México: Fondo de Cultura Económica.

Dennet, D. (2007). Neuroscience and Philosophy: Brain, Mind, and Language, Columbia University Press

Eco, U. (1979). Prólogo, en Gianni Castegnoli, 80's xerochromes, Milán: ediciones Franco Maria Ricci.

Even-Zohar, I. (1990). Polysystem Studies, Poetics Today, 1(11) 
Goodman, D. (1994). The Republic of Letters: A Cultural History of the French Enlightenment. Ithaca NY: Cornell University Pres

Habermas, J. (1996). Textos y contextos. Barcelona: Ariel

Holland, D. y Cole, M. (1995).Between discourse and schema: Reformulating a cultural-historical approach to culture and mind. Anthropology \& Education Quarterly, 26.

Jenkins, H. (2008). Convergence culture: la cultura de la convergencia de los medios de comunicación. Barcelona: Paidós

Jouvé, J.R. (2005). Esclavos de la ciudad letrada, esclavitud, escritura y colonialismo en Lima (1650-1700). Lima: Instituto de Estudios Peruanos.

Link, D. (1997). Literaturas comparadas, estudios culturales y análisis textual: por una pedagogía. Filología, 1(2), 5-14

López Maguiña, S., Portocarrero G., Silva R. y Ubilluz, J.C., (Eds.) (2007). Industrias Culturales. Máquina de Deseos en el Mundo Contemporáneo. Lima: Red para el Desarrollo de las Ciencias Sociales en el Perú

Morse, M. (1998). Virtualities: Television, Media Art, and Cyberculture (Theories of Contemporary Culture). Indiana: Univ. Press.

Sefton-Green, J., (Ed.) (1998): Digital Diversions: Youth Culture in the Age of Multimedia. UCL Press.

Sloterdijk, P. (2003). Crítica de la razón cínica. Madrid: Siruela

Verdú, V. (2003). El estilo del mundo. La vida en el capitalismo de ficción. Barcelona: Anagrama.

(Artículo recibido: 07-05-2010; revisado: 13-06-2010; aceptado: 19-06-2010) 